\title{
Combination use of
} intravascular ultrasound and dual-Iumen microcatheter guide for chronic total occlusion guidewire manipulation; $A$ practical method

\begin{abstract}
Aim: To investigate the effectiveness of a new guidewire (GW) manipulation technique of combination of Intravascular Ultrasonography (IVUS) and dual-lumen microcatheter guidance.

Methods: A 67 years old man presented with stump-less Chronic Total Occlusion (CTO) lesion in proximal part of Left Anterior Descending (LAD) coronary. By the combination use of IVUS and a dual-lumen microcatheter guide GW manipulation, GW successfully crossed the lesion. The efficacy was evaluated by the CTO model. Test 1 was used to determine which catheter is more suitable to obtain support force for penetration: the conventional IVUS-guide with single-lumen microcatheter or new IVUS-guide with dual-lumen microcatheter. Bench test 2 compared the proximity between CTO GW and IVUS catheter with conventional IVUS-guide and the new technique. Conventional IVUS guidance involved using a single-lumen microcatheter and IVUS cathetein, two separate GWs. The new technique involved using IVUS catheter and a dual-lumen microcatheter in the same GW. The CTO GW was manipulated through the over-the-wire lumen of the dual-lumen microcatheter.
\end{abstract}

Results: In test 1, the dual lumen microcatheter supported successful GW penetration, whereas the single lumen did not. In test 2, the CTO GW was manipulated more proximally to the IVUS catheter with the new technique than with conventional IVUS guidance.

Conclusion: The new technique is practical, as it provides more support force and more proximal visualisation than conventional IVUS guidance.

Keywords: Chronic total occlusion, intravascular ultrasound, bifurcated lesion, percutaneous coronary intervention, guidewire

\section{Introduction}

Chronic Total Occlusions (CTOs) in coronary arteries are found in about $20 \%$ of patients with coronary artery disease [1]. CTO is one of the challenging lesions associated with a lower success rate and higher prevalence of complications than other lesions [2]. The introduction of new devices and procedures significantly improved the success rate of Percutaneous Coronary Intervention (PCI) for CTO [3]. Recently, a treatment algorithm of PCI for CTO was introduced with the aim to serve as the basis for CTO guidewire $(\mathrm{GW})$ manipulation [4]. The algorithm recommends the use of Intravascular Ultrasonography (IVUS) guidance to penetrate the stump-less CTO [4].
Conventional GW manipulation involves use of a single-lumen microcatheter and it supports GW manipulation under IVUS-guide with two separate GWs [5]. A new GW manipulation technique involves a combination of IVUS and a dual-lumen microcatheter placed on the same GW guidance GW manipulation [6]. Although the technique has been just introduced, the efficacy of new IVUS-guide GW manipulation has not been fully elucidated. Therefore, the present study aimed to clarify whether the new GW manipulation technique is more practical than conventional IVUS-guide.

\section{A Case Report}

A 64 years old man complaining of
Hidenori Komiyama ${ }^{1 *}$, Masamichi Takano ${ }^{1}$, Tomoyo Miyakuni', Takeshi Ikeda ${ }^{1}$, Masato Matsushita ${ }^{1}$, Toru Inami ${ }^{1}$, Nobuaki Kobayashi ${ }^{1}$, Yasushi Miyauchi ${ }^{1}$, Yoshihiko Seino ${ }^{1}$, Wataru Shimizu ${ }^{2}$

${ }^{1}$ Cardiology, Nippon Medical School Chiba Hokusou Hospital, Chiba, Japan ${ }^{2}$ Cardiology, Nippon Medical School, Tokyo, Japan

*Author for correspondence:

takanom@nms.ac.jp 
exertional dyspnea admitted to our hospital. Coronary angiography showed Chronic Total Occlusion (CTO) in the Proximal Left Anterior Descending (LAD) coronary artery (Figure 1A). The lesion was stump-less. To confirm the CTO entry, intravascular ultrasound catheter (OptiCross $^{\mathrm{Tm}}$, Boston Scientific Corp., Natick, MA, USA) was placed in the nearest side branch to the CTO lesion [4]. A dual-lumen microcatheter (MC) (ASAHI SASUKE ${ }^{\circledast}$, Asahi Intecc, Tokyo, Japan) was inserted on the same IVUS mounted guidewire (GW) through rapid exchange (RX) lumen of dual-lumen MC, and a CTO GW (ASAHI Gaia First ${ }^{\oplus}$, Asahi Intecc) was manipulated through over-the-wire (OTW) lumen of dual-lumen MC [6]. The CTO-GW successfully crossed through CTO (Figures 1B$\mathrm{H})$ and the procedure was successful (Figures 1I,J).

\section{Methods}

\section{- Bench test 1}

Determining which is more suitable to obtain a satisfactory support force to penetrate the CTO in a vessel model: the single-lumen microcatheter or dual-lumen microcatheter. The CTO bifurcation model, which we have previously described, comprised a 5-mm main vessel and a 3-mm lateral branch vessel that branched at $90^{\circ}$ [7]. An agar plug was placed in the main vessel to mimic a CTO lesion just after the lateral vessel branches. Each microcatheter was positioned $10 \mathrm{~mm}$ distal from the occluded part (Figure 2A). A CTO GW was manipulated to penetrate the centre of the lesion. The GW was manipulated through the wire lumen of the single-lumen microcatheter. Then the GW was manipulated through the over-the-wire (OTW) lumen when the dual-lumen microcatheter was used.

\section{- Bench test 2}

Comparing the distance between the IVUS catheter (OptiCross ${ }^{\mathrm{TM}}$ coronary imaging catheter, Boston Scientific Corp., Natick, MA, USA) and CTO GW with conventional IVUS guidance and the new GW manipulation technique. The bifurcation model comprised a $5-\mathrm{mm}$ main branch and a $4.5-\mathrm{mm}$ daughter artery that branched at $50^{\circ}$ from a 4 - $\mathrm{mm}$ mother artery. The proximity between the IVUS catheter and GW was recorded.

\section{Results \\ Bench test 1: comparison of the support force}

The single-lumen microcatheter supported CTO GW manipulation contacted with the occluded part, but it did not penetrate the CTO (Figure 2B). The microcatheter was pushed back by the force of the GW. However, the duallumen microcatheter successfully penetrated the occluded part (Figure 2C). In dual-lumen microcatheter GW manipulation, a GW was placed in the lateral branch and inserted in the rapid exchange (RX) lumen of the duallumen microcatheter, and the CTO GW was manipulated through the OTW lumen of the

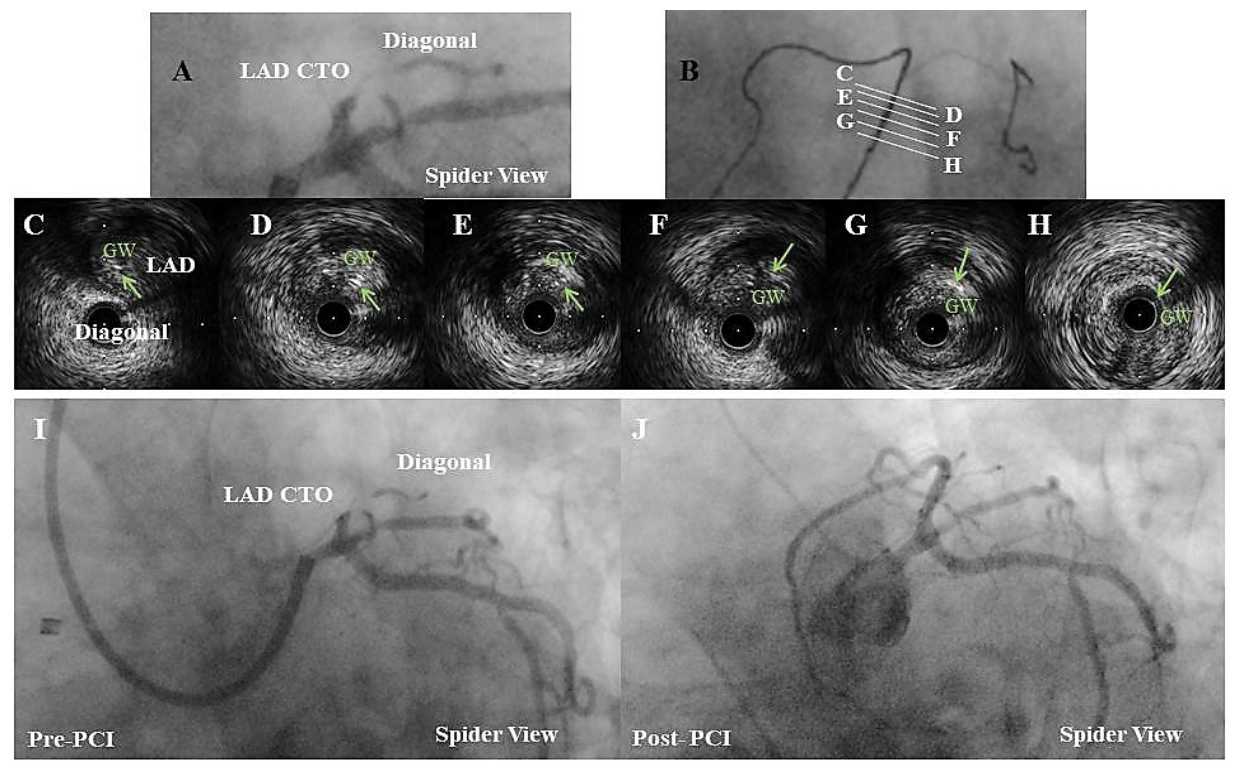

FIGURE 1: A case of proximal LAD CTO

Coronary angiography showed chronic total occlusion (CTO) in the proximal left anterior descending (LAD) coronary artery (Figure 1A). IVUS was placed in the diagonal branch nearest to the CTO lesion. A dual-lumen was inserted on the same IVUS mounted guidewire and a CTO-GW was manipulated through overthe-wire (OTW) lumen of dual-lumen MC. The CTO-GW successfully crossed through CTO (Figures 1B- H) and the procedure was successful (Figures 1I,J). 
dual-lumen microcatheter. The microcatheter was stabilised by the RX lumen (Figure 2C). In conventional IVUS guidance, the occluded lesion in the main branch was explored with the IVUS catheter placed in the lateral branch (Figure 2D). The GW did not penetrate the lesion, and the microcatheter was kicked back by the force of the GW (Figure 2D). Using the new GW manipulation technique, the IVUS catheter was placed in the lateral branch GW, and then the dual-lumen microcatheter was placed in the same GW through the RX lumen
(Figure 2E). The CTO-GW was manipulated through the OTW lumen of the dual-lumen microcatheter. The CTO GW successfully penetrated the lesion (Figure 2E).

\section{Bench test 2: comparison of the Proximity between the IVUS catheter and GW}

Using conventional IVUS guidance, the IVUS catheter and microcatheter were placed in separate GWs (Figure 3A). Using the new manipulation technique, the IVUS catheter and
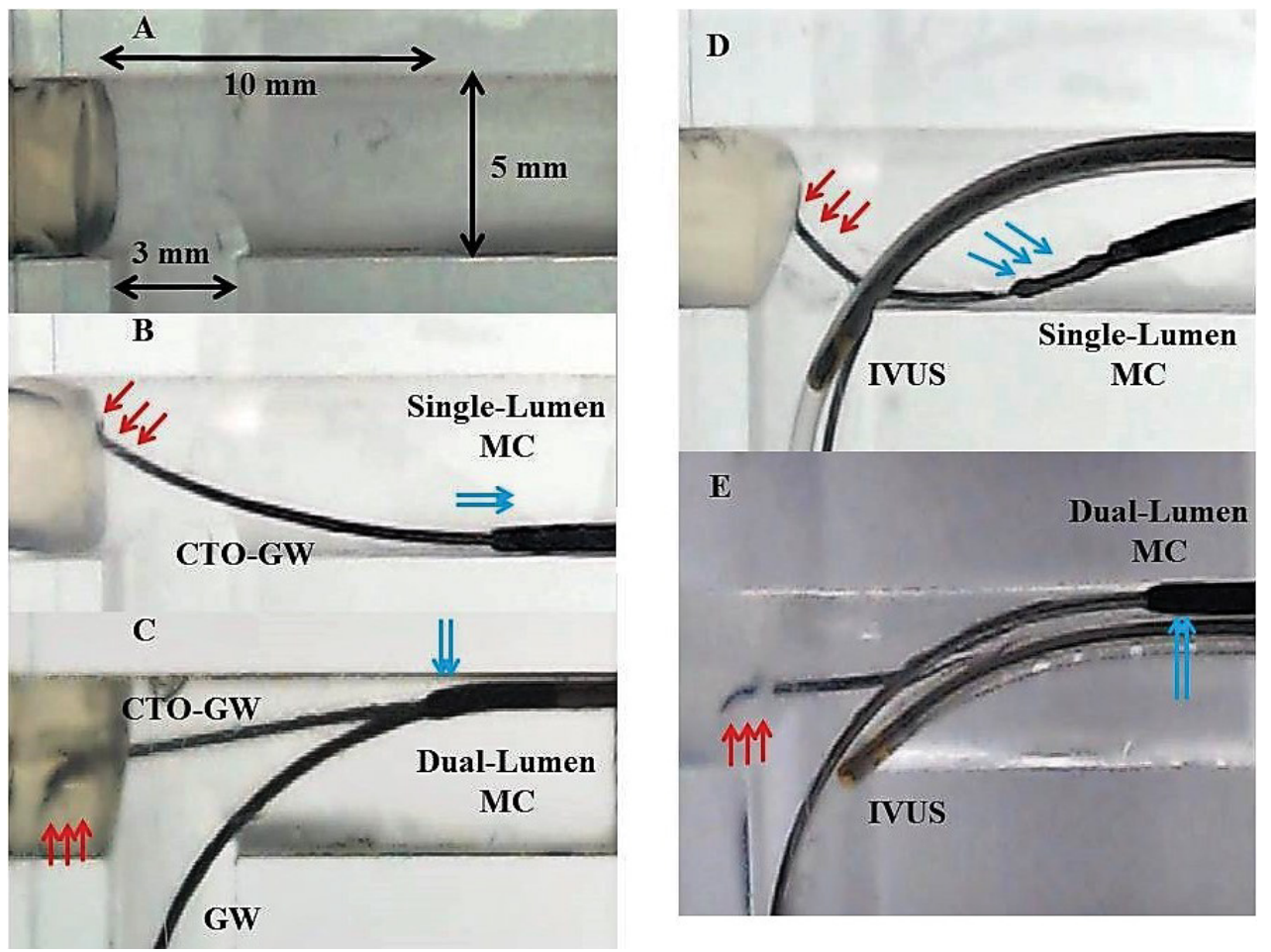

FIGURE 2. A. Vessel model mimicking chronic total occlusion (CTO).

The CTO bifurcation model is made of a 5- $\mathrm{mm}$ main vessel and 3-mm lateral branch vessel. An agar plug is inserted just after the branching lateral vessel. The microcatheter is positioned $10 \mathrm{~mm}$ distal from the occluded part.

B. The single-lumen microcatheter (Asahi Caravel ${ }^{\circ}$, Asahi Intecc, Tokyo, Japan) supports guidewire (GW) manipulation.

The CTO GW (Asahi Gaia Next 1) is manipulated in the single-lumen microcatheter (Asahi Caravel) to penetrate the centre of the occluded part, but the GW contacts the occluded part and does not penetrate the CTO. The microcatheter is pushed back by the force of the GW. The red arrowheads indicate the contact point of the GW. The blue arrowheads indicate the tip of the microcatheter.

C. The dual lumen microcatheter (Asahi Sasuke) supports GW manipulation.

A GW (Asahi SION Blue) is placed in the lateral branch and inserted into the rapid exchange (RX) lumen of the dual-lumen microcatheter. The microcatheter is stabilised by the RX lumen. The CTO GW is manipulated in the dual-lumen microcatheter through the over-the-wire (OTW) lumen. The CTO GW successfully penetrates the occluded part. The red arrowhead indicates the tip of the GW. The blue arrowheads indicate the tip of the microcatheter.

D. Conventional intravascular ultrasonography (IVUS) guidance for GW manipulation.

The SION Blue is placed in the lateral branch. The occluded lesion in the main branch is penetrated by the IVUS catheter (OptiCross coronary imaging catheter, Boston Scientific Corp., Natick, MA, USA) placed in the lateral branch. The GW does not penetrate the lesion. Note that the microcatheter is pushed back by the force of the GW. The red arrowheads indicate the contact point of the GW. The blue arrowheads indicate the tip of the microcatheter.

E. Combination of IVUS guidance and a dual-lumen microcatheter for GW manipulation.

The IVUS catheter is placed in the lateral branch GW, and then the dual-lumen catheter is placed in the same GW through the RX lumen. The CTO GW is manipulated through the OTW lumen of the dual-lumen microcatheter. The GW successfully penetrates the lesion. The red arrowhead indicates the tip of the guidewire. The blue arrowheads indicate the tip of the microcatheter. The microcatheter is stabilised by the RX lumen GW. 

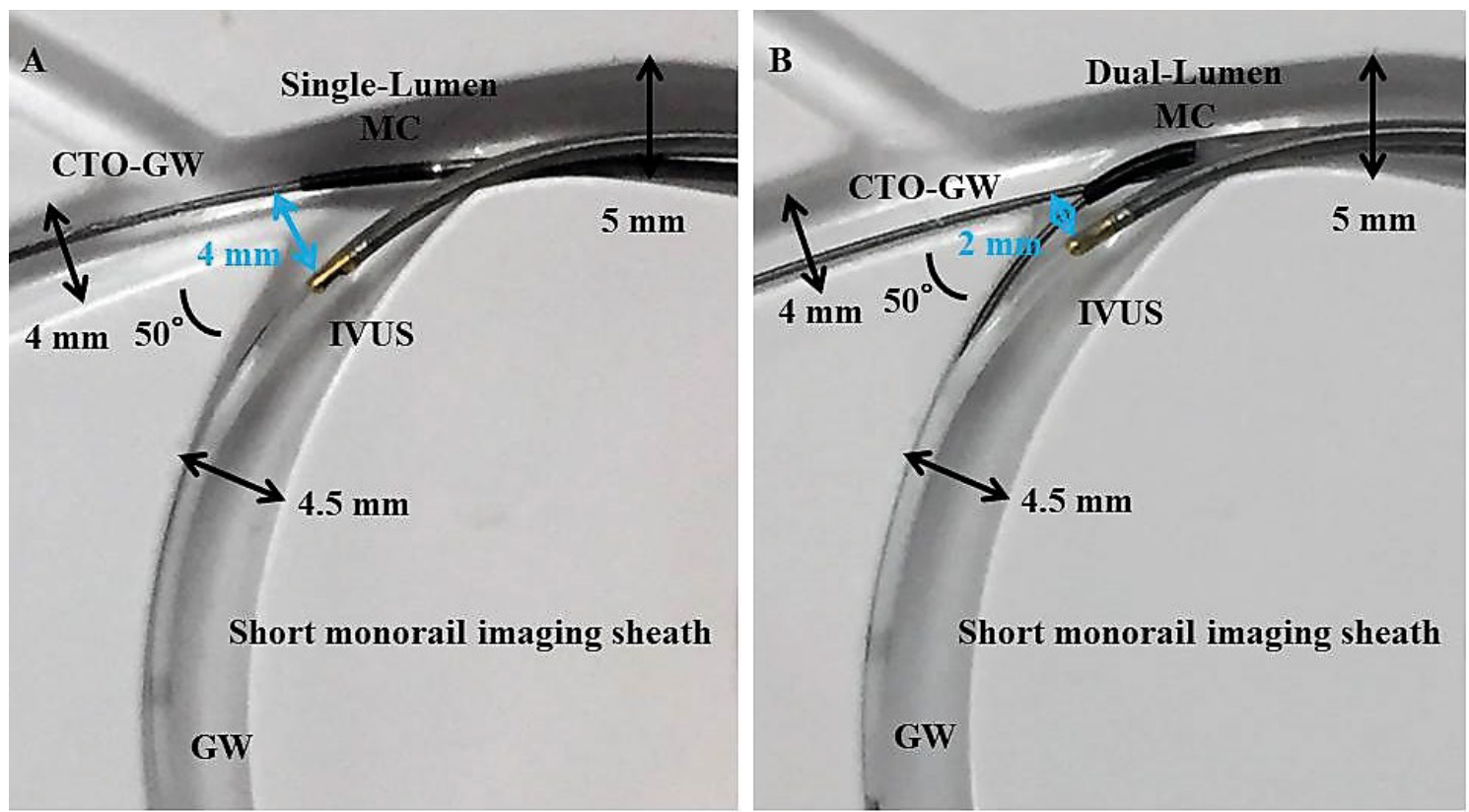

Figure 3. Comparison of the proximity of the intravascular ultrasonography (IVUS) catheter and guidewire (GW) between conventional IVUS guidance and the new GW manipulation technique.

The bifurcation model comprised a 5-mm main branch and a 4.5- $\mathrm{mm}$ daughter artery that branched at $50^{\circ}$ from a $4-\mathrm{mm}$ mother artery. In conventional IVUS guidance, the IVUS catheter and microcatheter are placed in separate GWs (A). The SION Blue is in the microcatheter, and the IVUS catheter is in the SION Blue. The SION Blue is manipulated in the single-lumen microcatheter under IVUS guidance (A). Using the new GW manipulation technique, the IVUS catheter and microcatheter are placed in the same guidewire (SION Blue) that is manipulated through the over-the-wire lumen of the dual-lumen microcatheter (B). The blue arrowheads indicate the distance between the IVUS catheter and manipulated GW. The distance between the IVUS catheter and manipulated GW is much closer with the new GW manipulation technique $(2 \mathrm{~mm})$ than with conventional IVUS guidance $(4 \mathrm{~mm})$.

microcatheter were placed in the same GW, and the CTO-GW was manipulated through the OTW lumen of the dual-lumen microcatheter (Figure 3B). The distance between the IVUS catheter and CTO GW was much closer with the new manipulation technique $(2 \mathrm{~mm})$ than with conventional IVUS guidance $(4 \mathrm{~mm})$.

\section{Discussion}

The new GW manipulation technique provides the CTO-GW with more support force for penetration, and more proximal positioning between the IVUS catheter and GW enables direct visualisation of the CTO entry and GW than conventional IVUS guidance. The standardised algorithm introduced by the Asia Pacific CTO Club plainly expresses the complex CTO procedures to interventionalists [4]. According to the algorithm, antegrade preparation is used first for which IVUS guidance is recommended to overcome the proximal CTO cap ambiguity [4]. The IVUS catheter should be placed in the nearest side branch of the CTO cap region if it exists to allow safe and successful GW manipulation [4]. Previous studies have shown the safety of penetration with IVUS guidance and stiff, tapered GWs, but the risk of GW perforation still exits [8]. The combination of IVUS guidance and a dual-lumen catheter for GW manipulation was first described by Kinoshita $e t$ al. [6]. The use of a dual-lumen microcatheter provides the CTO GW with sufficient support force for penetration, which is safer than the conventional wire escalation strategy. If a soft CTO GW with a lower tip load can penetrate the CTO lesions, its trackability in the CTO body is expected compared to a stiff CTO GW with a higher tip load. Herein, because the distance between the IVUS catheter and CTO GW was much closer with the new GW manipulation technique than with conventional IVUS guidance, interventionalist will be able to recognise the position of the GW more easily. Moreover, the IVUS catheter is stabilised by the GW placed in the side branch on the dual-lumen microcatheter, which facilitates exploration of the CTO cap and decreases the risk of perforation.

The main reason for an unsuccessful CTO procedure is failure of $\mathrm{GW}$ crossing, so GW 
crossing techniques have been developed to overcome this issue [9]. Although subintimal GW manipulation may be inevitable and allowed in CTO procedures [4,10], the ideal GW manipulation is crossing through the true lumen of CTO lesion and finishing the procedure without compromising any side branches. In the clinical setting, the GW can cross the subintima and be suboptimal. Antegrade GW manipulation can compromise the side branch if the GW crosses the subintimal space of the bifurcated region of the CTO. Retrograde GW manipulation can be considered in the contemporary algorithm. Even if the technology of the GW is improved [11], directly crossing the CTO lesion from the retrograde GW over the subintimal space can compromise the side branch. With the new GW manipulation technique, placing the IVUS in the side branch and manipulating the CTO GW with the dual-lumen microcatheter has an advantage in that it does not compromise the side branch because GWs coexist in the main branch and side branch.

There are some limitations to this study. First, because this study was only performed as an in vitro evaluation, the behaviours of the GWs in a human coronary artery may be different. Plaque distribution and calcification in a real human vessel may affect the behaviours of the GW and cause IVUS to be less effective. The space in the guiding catheter is limited to accommodate for simultaneous use of the IVUS catheter and dual-lumen microcatheter. Therefore, a slender IVUS catheter and dual-lumen microcatheter is needed to facilitate the new GW manipulation technique. Second, we only tested the first choice of GWs for placement in the side branch; more supportive wire might provide additional support force or change the position of the IVUS catheter and GW, which may facilitate the procedure. Lastly, we also tested only one kind of dual-lumen microcatheter; thus, using another kind of dual-lumen microcatheter may change the path of the GWs.

\section{Conclusion}

Compared with conventional IVUS guidance, a combination of IVUS guidance and a dual-lumen microcatheter is practical as it offers more support force and more proximal visualization to GWs.

\section{Acknowledgments}

We thank Akihisa Nakagawa (Asahi Intecc) and Masato Nakagawa (Boston Scientific) for providing valuable technical support and supplying the devices.

\section{Conflict Of Interest Statement}

The authors declare no conflicts of interest. 


\section{References}

Fefer P, Knudtson ML, Cheema AN, et al. Current perspectives on coronary chronic total occlusions the Canadian Multicenter Chronic Total Occlusions Registry. J Am Coll Cardiol. 59(11), 991997 (2012).

Movahed MR. Very high perforation rate in patients undergoing unsuccessful percutaneous coronary interventions of chronic total occlusions could explain worse outcome in these patients and not chronically occluded artery. JACC Cardiovasc Interv. 5(1), 116 (2012).

Suzuki Y, Tsuchikane E, Katoh O, et al. Outcomes of percutaneous coronary interventions for chronic total occlusion performed by highly experienced Japanese specialists: the first report from the Japanese CTO-PCI Expert registry. JACC Cardiovasc Interv. 10(21), 21442154 (2017).

Harding SA, Wu EB, Lo S, et al. A new algorithm for crossing chronic total occlusions from the Asia Pacific Chronic Total Occlusion Club. JACC Cardiovasc Interv. 10(21), 2135-2143 (2017).

Matsubara T, Murata A, Kanyama H, et al. IVUS - guided wiring technique: Promising approach for the chronic total occlusion. Catheter Cardiovasc Interv. 61(3), 381-386 (2004).

Kinoshita Y, Fujiwara H, Suzuki T. "Slipstream technique"-New concept of intravascular ultrasound guided wiring technique with double lumen catheter in the treatment of coronary total occlusions. JCC. 16(2), 52-55 (2017).

Komiyama H. In vitro evaluation of the appropriate guidewire for performing the reversed guidewire technique to treat severely angulated bifurcated lesions. www asiaintervention org. 58 (2017).

Mitsudo K, Yamashita T, Asakura Y, et al. Recanalization strategy for chronic total occlusions with tapered and stiff- tip guidewire. The results of CTO new techniQUE for STandard procedure (CONQUEST) trial. I Invasive Cardiol. 20(11), 571-577 (2008).

Matsuno S, Tsuchikane E, Harding SA, et al. Overview and proposed terminology for the reverse controlled antegrade and retrograde tracking (reverse CART) techniques. EuroIntervention: journal of EuroPCR in collaboration with the Working Group on Interventional Cardiology of the Eur. Heart J. 14(1), 94101 (2018).

Brilakis ES, Grantham JA, Rinfret $S$, et al. A percutaneous treatment algorithm for crossing coronary chronic total occlusions. JACC Cardiovasc Interv. 5(4), 367-379 (2012).

Khalili H, Vo MN, Brilakis ES. Initial Experience with the Gaia Composite Core Guidewires in Coronary Chronic Total Occlusion Crossing. I Invasive Cardiol. 28(2):22-25 (2016). 